\title{
Development of a low-cost electronic stethoscope - DIY Digital Stethoscope
}

Authors

Agam Jain ${ }^{1^{*}}$

Roshan Sahu ${ }^{2}$

Arohi Jain ${ }^{3}$

Thomas Gaumnitz ${ }^{3}$

Rakesh Lodha ${ }^{1}$

${ }^{1}$ Department of Pediatrics and ${ }^{2}$ Department of Neurosurgery, All India Institute of Medical Sciences, New Delhi ${ }^{3}$ Independent Researcher

*Corresponding author: Address: Hostel No. 8, Room No. 107, All India Institute of Medical Sciences, New Delhi- 110029, India. Mobile: +91-9999456533 Email: agamjains@gmail.com 


\title{
Development of a low-cost electronic stethoscope - DIY Digital Stethoscope
}

\begin{abstract}
Auscultation while wearing PPE (especially coveralls), using traditional stethoscopes is often not feasible. While some commercially electronic stethoscopes can circumvent this problem by playing audio using headphones over/ inside PPE, the currently available ones are too costly. We plugged an electret microphone into earpiece of a stethoscope and through various audio processing algorithms improved the audio quality. We packaged these processes into an app, freely available for download from Google Play Store. Audio quality through this device is at least equivalent to the one heard through traditional stethoscopes. We are validating the device in pediatric and adult clinical setup.
\end{abstract}

\section{Introduction}

COVID-19 pandemic has presented us with unique challenges. One of them is evaluating patients with lung pathologies, while wearing PPE. Wearing PPE (especially coveralls), because of covering of ears, precludes use of one of the time-tested tools of patient assessment - the stethoscope. Because of this, rapid assessment of patients at multiple points of time is affected. To overcome this, use of bedside ultrasonography has been advocated (1); however, ultrasonography is costly and requires additional training. Also, the availability of ultrasonography is not universal. Besides, there is no easy alternative for detection of wheeze and evaluation of symmetry of breath sounds, both of which have implications for interventions. One of the ways around, is to use electronic stethoscopes, but they are too costly for mass deployment, with a price averaging around $500 \$$ from established brands $(2,3)$. Here we provide a simple, cost-effective method to convert an existing stethoscope into a digital one: DIY (Do It Yourself) Digital Stethoscope.

\section{Materials and Methods:}

A stethoscope requires a sensor to collect the sounds, a processor and a playback system.

We tried various permutations and combination of sensors (electret vs Micro-electroMechanical System [MEMS] vs piezoelectric) in different configurations (directly applied to skin vs within the chest-piece vs within the tubing of the stethoscope vs at the ear tip). For our model, we decided to use an electret microphone (e.g. collar mic) at the ear tip. The objective of processing was to amplify the signal and reduce the noise. During preliminary testing, we captured audio directly into an open source, free license software called Audacity $\otimes$ (Audacity, US) version 2.4.2 running on a computer. Thereafter, we compared multiple audio processing algorithm for each above said function, in different combinations, determined the optimum settings and we decided on the following sequence: Noise Reduction, Low pass filter, Compressor, Amplification with Noise Floor. We also developed a dedicated cardiac mode with narrower low pass filter to improve the 
clarity. These processes were adapted to the DIY stethoscope app for automated use on a mobile phone.

\section{Materials required:}

I. Any traditional stethoscope

II. Compliant Earpiece with large opening: comes with stethoscope

III. Electret microphone

IV. Android based smartphone

V. 'DIY Digital Stethoscope' app available on Google Play Store

VI. Playback system: Bluetooth Headphones/ earphones

\section{Device Assembly Protocol:}

1. Select an earpiece which has a large opening and is elastic enough.

2. Remove the foam windscreen from the microphone.

3. Fit the microphone into the earpiece, ensure a snug fit.

4. Plug the jack from the microphone into the smartphone (Figure 1).

5. Open the DIY stethoscope app.

6. Pair your headphones to the smartphone.

7. Hold the chest piece gently to the area you want to auscultate

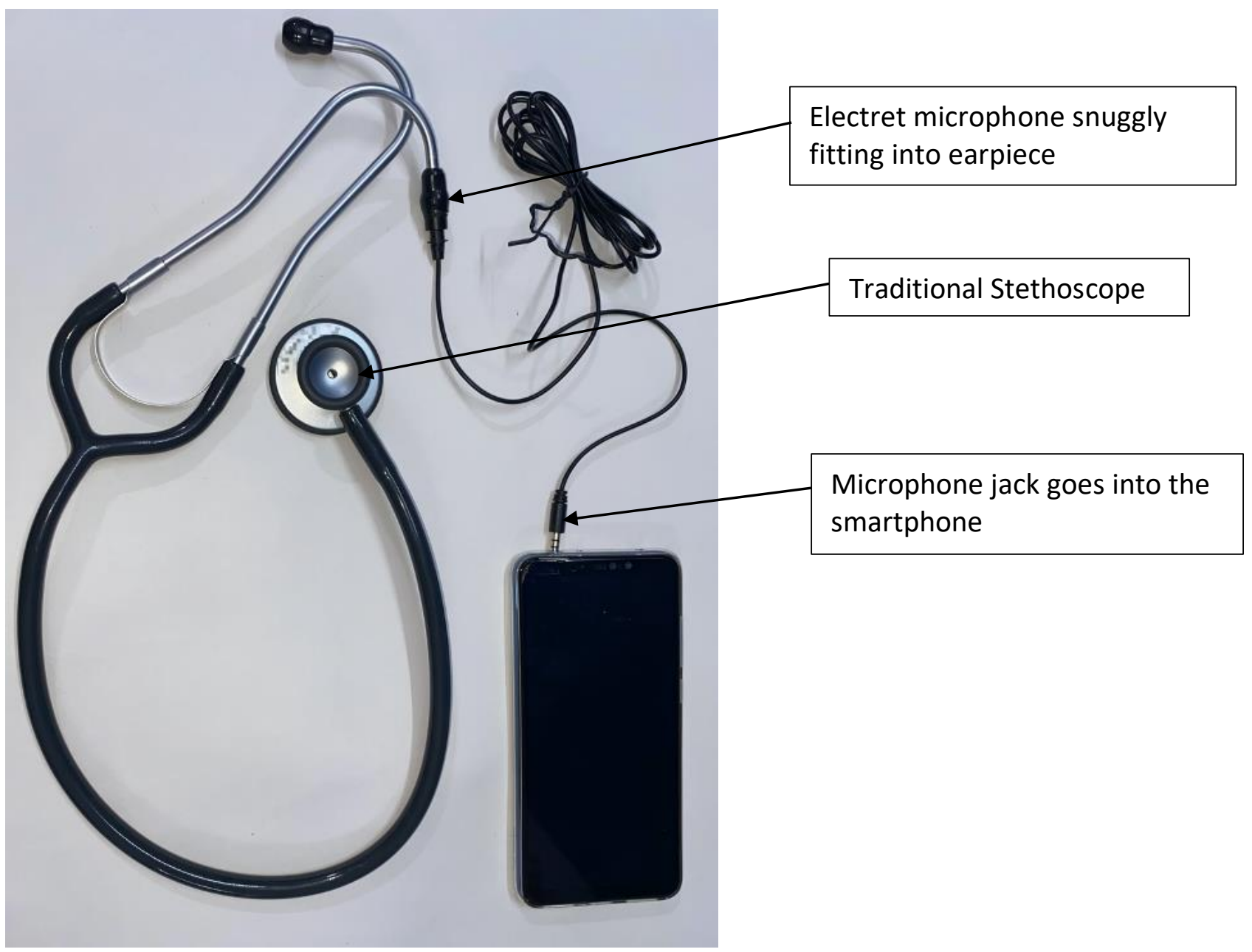

Figure 1: Device assembly showing the stethoscope, microphone and the mobile phone 
The device can also be used in following configuration (Figure 2) :

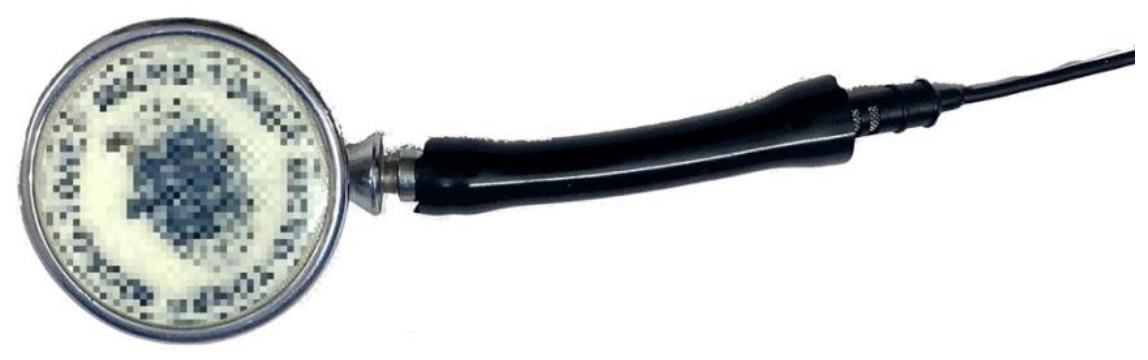

Figure 2: Microphone attached at chest piece

There are no standardized techniques to test the output of a stethoscope. We tested the device on ourselves for both breath and cardiac sounds in both silent as well as noisy environment. We also calculated the lag created by the system, by playing a pure tone and determined the time it takes for playback to start, using a digital stopwatch.

\section{Results}

Through the device (Figure 1) we were able to auscultate breath sounds as well as cardiac sounds with good clarity and amplitude which were qualitatively similar to a normal stethoscope. The device reduced noise to an extent that it didn't interfere in auscultation. However, loud, irregular background sounds could still be heard similar to that of a traditional stethoscope. There is a lag in playback of about $0.7 \mathrm{~s}$

\section{Discussion}

We took a modular approach for our device, taking advantage of the fact that most healthcare workers, already possess good quality components required for an electronic stethoscope (e.g. high-end stethoscope, a smartphone and headphones). They may need to buy, only the microphone, which is inexpensive.

We chose to use an electret microphone (e.g. collar mic) for our device because of low cost, wide availability and easy interface with the smartphone. The sensor can be placed directly on the skin but pairing it with the chest-piece of a stethoscope takes advantage of it's bigger collecting surface. The design and quality of chest-piece and tubing of the stethoscope has an impact on the audio output, with efficiency improving with smaller length and diameter of tubing (4). The microphone, could be placed anywhere from the chest-piece, to within the tubing or at the earpiece. The advantage of placing the microphone at the earpiece is that it doesn't require sacrificing the stethoscope, and it can be restored back to its original state. So, one can take advantage of better acoustics of high-end stethoscopes which user already may possess, without worrying about the cost. The microphone should fit snugly into the earpiece, not allowing any leakage of sound around it. In preliminary testing, placing the sensor at earpiece, allowed interference from the ambient sound, entering 
through the 'open' earpiece. Blocking it or placing sound absorbing material resulted in interference with the signal captured at the sensor probably as a result of signal from the 'open' earpiece being reflected back towards the stethoscope and interfering with the signal. However, some amount of noise control can be done at processing stage. So, we chose to keep the ear-piece open.

Removal of ambient noise and other artifacts such as motion artifact is a challenging task, with some commercially available products using active noise cancellation (5), Adaptive Line Enhancement (ALE) or Least Mean Squared or other Machine Learning Protocols, with varying degrees of success (5), (6).

We chose not to use them, as simultaneous playback of the captured sound was required and these are computationally taxing algorithm and may not work very well with wide range of processing capacities in different mobile phones. Also, these algorithm work by averaging multiple samples of signal collected over period of time, so any finding that occurs occasionally, runs the risk of being lost through these processing algorithms. We used "Noise Reduction" feature of Audacity ${ }^{\circledR}$, which collects a sample of ambient sound, before signal is collected and then analyses intensity of sound at different frequencies and whenever for a given frequency the intensity of sound doesn't vary significantly from that of the ambient sound, the intensity of sound in that frequency is reduced. Movement of diaphragm of stethoscope over skin causes a motion artifact, which cannot be eliminated completely with the above said protocols. Based on preliminary testing, holding the chest piece gently over the skin produces less motion artifact as compared to pressing deeply.

The audio output after processing can be played through an externally connected speaker, inbuilt speaker of the mobile, earphones, headphones etc. However, since the signal consists of sounds mainly in low frequency range, devices capable of sound reproduction in this range, i.e. high bass, offer better output. Headphones offer ease of wearing, comfortable wear and ability to transmit sound while wearing PPE. Playback of sound through speaker, during auscultation, may cause an audio loop with same sound being fed forward to sensor and being amplified repeatedly, however they may be excellent choice for training.

This device offers a low-cost solution to one of the challenges faced in managing COVID-19 patients. It can be rapidly deployed and can have an impact on patient care, not only in inpatient settings but also in outpatient settings where, doctors can auscultate the patient from a safe distance. This device can also be used for tele-medicine where healthcare workers at remote settings can help doctor auscultate the patient and make a more informed decision. The device allows multiple users to auscultate simultaneously by playback through a speaker, which can be used in medical education. The device can also help healthcare professionals with hearing deficits.

Amongst the configurations used, microphone at earpiece doesn't harm the stethoscope and it can be reused afterwards. However, the design also allows to salvage any old/broken stethoscope with intact chest-piece (Figure 2).

Compared to other electronic stethoscope, this device provides similar functionalities such as sound amplification, noise reduction, ability to share auscultated sounds but is cheaper, doesn't need to be charged and there are no limitation to the length or number of recordings that can be made. 
The device has not yet been validated formally in clinical setting and a follow-up study is planned comparing it to auscultation using traditional stethoscope alone. Although the processing does a good job at reducing noise, however, all the noise cannot be filtered without harming the signal. So, noise reduction at source, such as muting any unnecessary alarm will improve the auscultation quality. The quality of auscultation also depends on the stethoscope used, with lower quality stethoscopes distorting the sound. There is also a lag in playback, so situations requiring coordination between auscultation and another process such as manual blood pressure measurement may require more careful measurement or alternative method.

\section{Conclusions}

Here, we present a technique to convert a stethoscope to an electronic one, aimed at improving patient care while wearing PPE. In preliminary evaluation, the quality of auscultation is acceptable. The device could also be used for training of students in medical and nursing colleges. The device still needs to be validated in a clinical setup.

Financial Support: None

Competing Interest: None

\section{Contribution}

AJ was involved in conceptualization, development and testing of the device, wrote the paper

RL was involved in conceptualization, development and testing of the device, contributed to writing the manuscript

RS was involved in conceptualization, development and testing of the device, contributed to writing the manuscript

$\mathrm{AJ}^{3} \& \mathrm{TG}^{3}$ developed the app, contributed to writing the manuscript

\section{References}

1. Buonsenso D, Pata D, Chiaretti A. COVID-19 outbreak: less stethoscope, more ultrasound. Lancet Respir Med. 2020 May;8(5):e27.

2. $3 M$ Littmann Electronic Stethoscope 3200 [Internet]. USA Medical and Surgical Supplies. [cited 2020 Sep 8]. Available from: https://www.usamedicalsurgical.com/3m-littmannelectronic-stethoscope-3200/

3. Thinklabs One - Digital Stethoscope [Internet]. Thinklabs. [cited 2020 Sep 8]. Available from: https://store.thinklabs.com/products/thinklabs-one-digital-stethoscope

4. Rappaport MB, Sprague HB. The effects of tubing bore on stethoscope efficiency. Am Heart J. 1951 Oct 1;42(4):605-9. 
5. Leng S, Tan RS, Chai KTC, Wang C, Ghista D, Zhong L. The electronic stethoscope. Biomed Eng OnLine [Internet]. 2015 Jul 10 [cited 2020 Aug 17];14. Available from: https://www.ncbi.nlm.nih.gov/pmc/articles/PMC4496820/

6. Lakhe A, Sodhi I, Warrier J, Sinha V. Development of digital stethoscope for telemedicine. J Med Eng Technol. 2016 Jan 5;40(1):20-4. 